This discovery implies that the differentiation of $B$ cells in the spleen may be enhanced by acetylcholine. Indeed, the authors demonstrate the expression of nicotinic acetylcholine receptor (nAChR) subunits by spleen $B$ cells. To examine the contribution of acetylcholine signalling to B cell responses, they transplanted bone marrow cells from animals in which the $\alpha 9$ nAChR subunit gene was deleted into irradiated mice. In these reconstituted animals, all B cells lacked $\alpha 9$-containing nAChRs. The capacity of these animals to produce SPPCs following NP-KLH immunization was impaired, indicating that splenic nerve activity enhances SPPC production through the activation of $\mathrm{B}$ cell $\mathrm{nAChRs}$.

Many autonomic responses are subject to top-down modulation by the brain. Through viral-mediated retrograde tracing, optogenetic stimulation and electrophysiological recordings, the authors showed that the splenic nerve is functionally connected to corticotrophin-releasing hormone $(\mathrm{CRH})$-producing neurons originating in two particular forebrain areas known to be involved

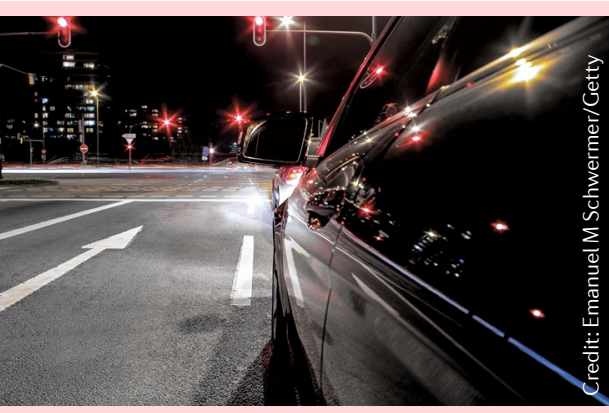

The authors previously found that S276 phosphorylation of AQP4 is required but not sufficient for its subcellular relocalization to the plasma membrane. Thus, they examined whether $\mathrm{CaM}$ influences this process independently to its effects on PKA. They identified a putative CaM binding site in the carboxyl terminus of AQP4 and showed that the two proteins could bind via this site. Moreover, in a human cell line, hypotonicity triggered the relocalziation of wild-type AQP4 but not a mutant form of AQP4 in which the CaM binding site was disrupted, indicating that the AQP4-CaM interaction is key for the rapid relocalization of AQP4.

The authors next examined whether targeting AQP4 localization improves in the body's responses to stress and threats - the central amygdala (CeA) and the paraventricular nucleus (PVN) of the hypothalamus. Genetic ablation or pharmacogenetic inhibition of these neurons impaired SPPC formation after immunization, whereas activation of the neurons boosted the number of SPPCs produced.

These findings prompted the authors to ask whether tasks that activate the CeA or PVN can modulate adaptive immune responses. They discovered that 4 days of exposure to mild stress (by placing the mice on an elevated platform for 3 minutes) activated CeA and PVN CRH neurons and stimulated the production of SPPCs after immunization.

This study provides evidence for a direct pathway through which brain activity can tune immune system functioning, adding to our growing understanding of brain-body interconnectivity.

\section{Katherine Whalley}

ORIGINAL ARTICLE Zhang, X. et al. Brain control of humoral immune responses amenable to behavioural modulation. Nature https://doi.org/ 10.1038/s41586-020-2235-7 (2020)

functional recovery in the rat $\mathrm{SCl}$ model. Treatment with trifluoperazine, a CaM inhibitor licensed for use in humans, or a PKA inhibitor directly after injury improved signal conduction across the lesion site over the first 6 weeks after injury. Moreover, treated animals took less time to remove tape adhered to their hindpaw (a test of sensory function) than untreated animals 1 week after injury and showed normal performance levels by 3 weeks. Similarly, treated animals showed improvements in a ladder-crossing task (a motor task) 1 week after injury and showed near-normal performance another week later, but untreated animals showed no improvements. Together, these findings indicate that, in rats, treating CNS oedema immediately after $\mathrm{SCl}$ with drugs that acutely affect $\mathrm{APQ} 4$ subcellular localization improves sensory and motor function.

\section{Darran Yates}

ORIGINAL ARTICLE Kitchen, P. et al. Targeting aquaporin-4 subcellular localization to treat central nervous system edema. Cell 181, 784-799 (2020)

\section{IN BRIEF}

\section{$\Rightarrow$ NEUROIMAGING}

\section{Figuring out body integrity dysphoria}

Individuals with body integrity dysphoria (BID) feel as if they do not own one of their limbs, and seek its amputation. Saetta et al. used MRI to explore functional and structural differences in the cortex of 16 men with BID targeting the left leg. In these individuals, the sensorimotor area corresponding to the left leg showed reduced functional connectivity, and the left premotor cortex, which integrates multimodal sensory information, was atrophic. Moreover, the right superior parietal lobule, which is known to represent body image, showed functional and structural abnormalities, with its atrophy correlating with strength of desire for amputation.

ORIGINAL ARTICLE Saetta, G. et al. Neural correlates of body integrity dysphoria. Curr. Biol. https://doi.org/10.1016/j.cub.2020.04.001 (2020)

\section{BRAIN EVOLUTION}

\section{Audition for a speaking role}

The human arcuate fasciculus (AF) is a dorsal pathway crucial for language ability. Although chimpanzees have an AF homologue, whether monkeys have one too is controversial. Diffusion MRI data from humans, chimpanzees and macaques showed that, like chimpanzees, macaques have a homologous, bilateral connection from the auditory cortex to the inferior frontal cortex, in both hemispheres. These findings support the hypothesis that language arose through the evolution of primate auditory pathways.

ORIGINAL ARTICLE Balezeau, F. et al. Primate auditory prototype in the evolution of the arcuate fasciculus. Nat. Neurosci. 23, 611-614 (2020)

\section{VISION}

\section{Action pictures}

Visual cortical prostheses (VCPs) may be useful for many individuals with acquired blindness. VCP stimulation of a point on the visual cortex elicits the perception of a flash of light, known as a phosphene; however, phosphenes resulting from concurrent stimulation of multiple points on the visual cortex do not combine into a coherent shape. Here, the authors used a dynamic pattern of stimulation known as current steering, whereby the sequential delivery of current via two electrodes in turn leads to the formation of a 'virtual electrode' between them - resulting in perception of a line. Such stimulation led to the perception of letters by sighted and blind participants.

ORIGINAL ARTICLE Beauchamp, M. S. et al. Dynamic stimulation of visual cortex produces form vision in sighted and blind humans. Cell 181, 774-783 (2020)

\section{$\Rightarrow$ LEARNING AND MEMORY}

\section{A-to-Z interactions in fear extinction}

The functional importance of Z-DNA - a left-turning helical conformation of DNA - is unclear. Marshall et al. identified an immediate increase in the formation of Z-DNA in neurons in the prefrontal cortex (PFC) of mice that had undergone fear learning. Subsequent fear extinction training led to increases in the levels of the Z-DNA-binding protein ADAR1 in activated PFC neurons, where it bound to more than 100 Z-DNA loci and promoted RNA editing. ADAR1-Z-DNA binding reduced Z-DNA levels, and knock-down of Adar1 disrupted fear extinction learning, suggesting the interaction between Z-DNA and ADAR1 is necessary for memory flexibility. ORIGINAL ARTICLE Marshall, P. R. et al. Dynamic regulation of Z-DNA in the mouse prefrontal cortex by the RNA-editing enzyme Adar 1 is required for fear extinction. Nat. Neurosci. https://doi.org/10.1038/s41593-020-0627-5 (2020) 